\title{
Analyzing degradation effects of organic light-emitting diodes via transient optical and electrical measurements
}

Cite as: J. Appl. Phys. 117, 215502 (2015); https://doi.org/10.1063/1.4921829

Submitted: 11 March 2015. Accepted: 18 May 2015. Published Online: 02 June 2015

Tobias D. Schmidt, Lars Jäger, Yutaka Noguchi, Hisao Ishii, and Wolfgang Brütting
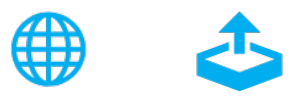

View Online

Export Citation

\section{ARTICLES YOU MAY BE INTERESTED IN}

Impedance spectroscopy as a probe for the degradation of organic light-emitting diodes Journal of Applied Physics 107, 054501 (2010); https://doi.org/10.1063/1.3294642

Operational degradation of organic light-emitting diodes: Mechanism and identification of chemical products

Journal of Applied Physics 101, 024512 (2007); https://doi.org/10.1063/1.2430922

Direct evidence for degradation of polaron excited states in organic light emitting diodes Journal of Applied Physics 105, 124514 (2009); https://doi.org/10.1063/1.3151689

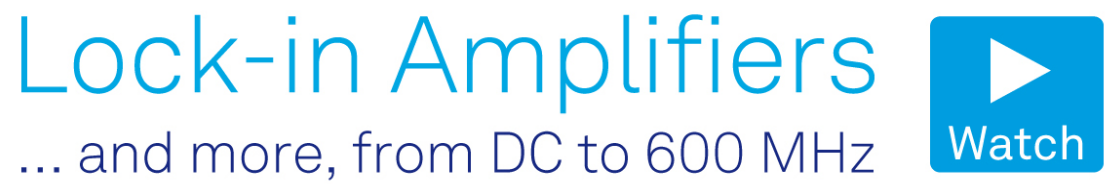




\title{
Analyzing degradation effects of organic light-emitting diodes via transient optical and electrical measurements
}

\author{
Tobias D. Schmidt, ${ }^{1, a)}$ Lars Jäger, ${ }^{1}$ Yutaka Noguchi, ${ }^{2,3}$ Hisao Ishii, ${ }^{3}$ \\ and Wolfgang Brütting ${ }^{1, b)}$ \\ ${ }^{1}$ Institute of Physics, University of Augsburg, Augsburg, Germany \\ ${ }^{2}$ Department of Electronics and Bioinformatics, School of Science and Technology, Meiji University, \\ Kawasaki, Japan \\ ${ }^{3}$ Center of Frontier Science, Chiba University, Chiba, Japan
}

(Received 11 March 2015; accepted 18 May 2015; published online 2 June 2015)

\begin{abstract}
Although the long-term stability of organic light-emitting diodes (OLEDs) under electrical operation made significant progress in recent years, the fundamental underlying mechanisms of the efficiency decrease during operation are not well understood. Hence, we present a comprehensive degradation study of an OLED structure comprising the well-known green phosphorescent emitter $\operatorname{Ir}(\mathrm{ppy})_{3}$. We use transient methods to analyze both electrical and optical changes during an accelerated aging protocol. Combining the results of displacement current measurements with time-resolved investigation of the excited states lifetimes of the emitter allows for a correlation of electrical (e.g., increase of the driving voltage due to trap formation) and optical (e.g., decrease of light-output) changes induced by degradation. Therewith, it is possible to identify two mechanisms resulting in the drop of the luminance: a decrease of the radiative quantum efficiency of the emitting system due to triplet-polaronquenching at trapped charge carriers and a modified charge carrier injection and transport, as well as trap-assisted non-radiative recombination resulting in a deterioration of the charge carrier balance of the device. (C) 2015 AIP Publishing LLC. [http://dx.doi.org/10.1063/1.4921829]
\end{abstract}

\section{INTRODUCTION}

Organic light-emitting diodes (OLEDs) found their way to daily life of people especially in terms of small area displays used in smartphones and tablet computers ${ }^{1}$ but also more and more in large area television screens ${ }^{2}$ and general lighting applications. ${ }^{3}$ The advantages of OLEDs are miscellaneous as it is, e.g., possible to fabricate very thin, plane light sources, which can even be flexible, with arbitrary shapes by simple solution or evaporation based techniques. The efficiency of state-of-the-art OLEDs can already compete with common fluorescent tubes; ${ }^{4}$ nevertheless, long-term stability of this technology is not yet satisfactory for general lighting applications as noticeable degradation takes place in not acceptable time scales for consumers. Typically, during electrical operation, the luminance of the OLED decreases with a simultaneous increase in the required voltage (constant current conditions). The underlying mechanisms of this behavior have been analyzed in the past by different techniques such as time-resolved photoluminescence measurements ${ }^{5-7}$ and impedance spectroscopy $^{8-10}$ (IS) to achieve information about the optical and electrical changes, respectively, during degradation. However, in previous investigations, rarely have both aspects been analyzed simultaneously, and hence a comprehensive understanding of the correlation between electrical and optical aging effects is still missing. In this article, we combine three different investigation methods during the degradation process. IS as well as displacement current measurements (DCM) are used to analyze electrical characteristics such as charge carrier injection,

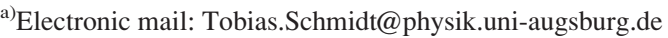

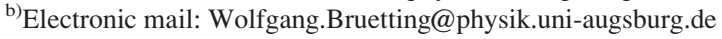

transport, and trap-formation, and time-resolved electroluminescence spectroscopy (TRELS) gives information about the excited states lifetime of the emitting molecules. With the information achieved via DCM and IS, it is possible to explain the changes of the non-radiative rate and therewith the emitter's radiative quantum efficiency exclusively by the creation of positively charged trap states in the emission layer (EML) via the triplet-polaron-quenching (TPQ) model modified for immobile charge carriers. Therewith, a comprehensive degradation analysis is presented identifying, correlating, and quantifying the underlying degradation process, namely, the creation of trap states in the EML directly causing a decreasing radiative quantum efficiency of the emitting system as well as losses of charge carrier balance due to, e.g., trap-assisted (nonradiative) recombination (TAR) and changes in charge carrier injection.

\section{DEVICE STRUCTURE AND MATERIALS}

In this work, we investigate the degradation behavior of a prototypical organic light-emitting diode via transient electrical and optical measurements. The stack layout of the device under investigation is shown in Fig. 1 and consists of a $0.7 \mathrm{~mm}$ glass substrate covered with a patterned ITO layer $(140 \mathrm{~nm})$ as anode, followed by a $15 \mathrm{~nm}$ thick hexa-azatriphenylene-hexanitrile (HATCN) film acting as hole injection layer (HIL) and $100 \mathrm{~nm}$ of N,N'-bis(1-naphthyl)-N, $\mathrm{N}^{\prime}$-diphenyl-1,1'-biphenyl-4, $4^{\prime}$-diamine $(\alpha-N P D)$ as hole transport layer (HTL). Subsequently, the $10 \mathrm{~nm}$ thick EML is formed by the matrix material 4,4'-bis(N-carbazolyl)-1, $1^{\prime}$-biphenyl (CBP) doped with $6.5 \mathrm{wt}$. \% of the green phosphorescent emitter fac-tris(2-phenylpyridine)iridium $\left(\operatorname{Ir}(\mathrm{ppy})_{3}\right)$. The 


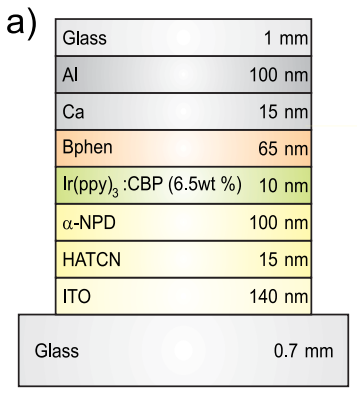

b)

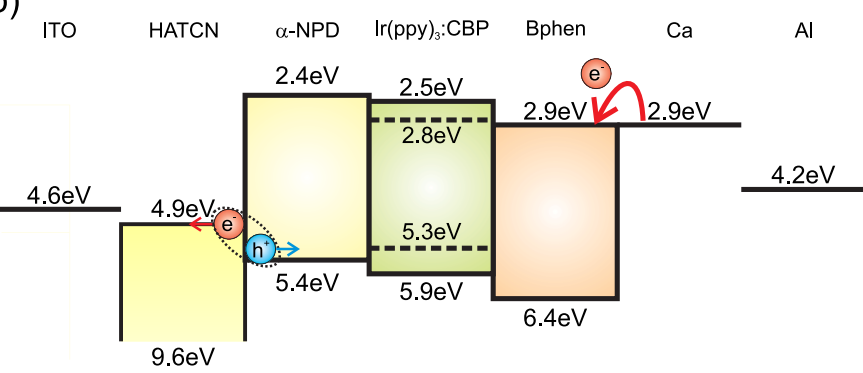

c)
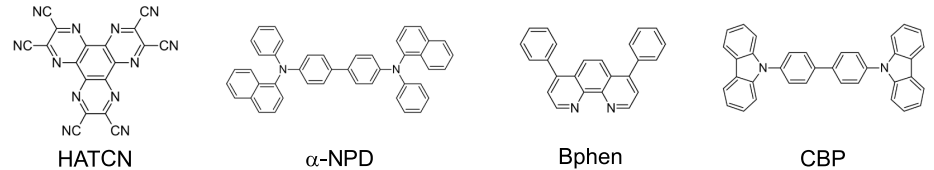

CBP

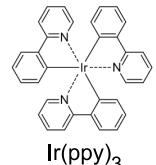

FIG. 1. (a) OLED stack layout under investigation. Strictly speaking, the encapsulation glass at the top of the device is not directly lying on the cathode of the OLED and therewith a little amount of the inert gas atmosphere of the glovebox is captured between the cathode and the glass lid. (b) Schematic energy diagram of the HOMO and LUMO levels of the used organic molecules. $^{11-15}$ (c) Molecular structure of the used organic materials.
EML is followed by a $65 \mathrm{~nm}$ thick electron transport layer (ETL) consisting of 4,7-diphenyl-1,10-phenanthroline (Bphen). Finally, a $15 \mathrm{~nm}$ thick calcium cathode was deposited, covered by $100 \mathrm{~nm}$ aluminum. The schematic energy diagram of HOMO and LUMO levels of the different organic layers shown in Fig. 1 (with energy levels are taken from Refs. 11-15) clearly illustrates the specific functions of the organic layers. Strictly speaking, HATCN is more acting as hole generation layer than as HIL. This material exhibits a very large energy gap of $4.7 \mathrm{eV}$, prohibiting the absorption of light that is generated in the emission layer of the device. Moreover, HATCN shows a very high electron affinity due to its deep lying LUMO energy level. Thus, electrons can easily travel from the HOMO of the adjacent hole transporting material into the LUMO of HATCN, creating holes in the $\alpha$-NPD layer. The electrons in the LUMO of HATCN are then traveling (due to the applied electric field) to the ITO anode and are extracted there. In order to protect the organic layers and the cathode from moisture and ambient air, the whole device was encapsulated in a nitrogen glovebox with a second glass lid connected to the bottom substrate by an epoxy adhesive. All organic materials were purchased from commercial suppliers in sublimed grade and have been evaporated together with the metals in an ultra-high vacuum chamber attached to the mentioned nitrogen glovebox without breaking the vacuum. The ITO covered glass substrate was cleaned by several solvents in ultrasonic baths and an UV-ozone treatment for several minutes in a clean room. Due to the small pixel size of $1 \mathrm{~mm}^{2}$ only, and the structured organic layers, the device under investigation exhibits a very short $R C$-time of only about $50 \mathrm{~ns}$. This low latency device structure allows for an analysis of the excited states lifetimes of the phosphorescent emitter by TRELS.

\section{METHODS}

\section{A. Time-resolved electroluminescence spectroscopy}

In order to measure the excited states lifetime of the emitting species inside the OLED structure for different degradation steps, time-resolved electroluminescence spectroscopy was performed. Therefore, a square-wave voltage pulse with variable height and length is applied to the OLED by an arbitrary waveform generator (Tabor Electronics
WW2571A). The voltage-pulse length is chosen such that steady state conditions of the EL signal are achieved. The phosphorescent response of the OLED is collected by a collimator lens coupled with a multi-mode glass fiber which carries the light emission to a grating spectrometer (Princeton Instruments Acton SpectraPro 2300i) attached to a streak camera system (Hamamatsu Photonics C5680) allowing for a simultaneous resolution in time and wavelength. Fitting the detected EL decay directly after switching off the applied voltage by a mono-exponential function results in the excited states lifetime of the phosphorescent emitter $\left(\operatorname{Ir}(\mathrm{ppy})_{3}\right)$ :

$$
I(t)=I_{0} \cdot e^{-t / \tau}+C
$$

with $\tau$ being the actual measured excited states lifetime and $C$ taking into account the background noise of the streak camera system. Note, that in the following TRELS investigations, moderate negative electrical bias is applied during the off state of the measurements in order to extract residual free charges to minimize their influence during the decay of the electroluminescence signal. ${ }^{16}$

The pristine (intrinsic) excited states lifetime in the OLED cavity $\left(\tau_{0}\right)$, without current flow and trapped charge carriers, is given by the radiative rate $\left(\Gamma_{\mathrm{r}}\right)$ modified by the so-called Purcell ${ }^{17}$ factor $F$ considering cavity effects, as well as by the non-radiative rate $\left(\Gamma_{\mathrm{nr}}\right)$ :

$$
\tau_{0}=\left(F \cdot \Gamma_{\mathrm{r}}+\Gamma_{\mathrm{nr}}\right)^{-1} .
$$

\section{B. Impedance spectroscopy}

In order to analyze the electrical characteristics of the OLEDs during electrical aging, impedance spectroscopy is performed. Therefore, a small alternating voltage $\left(V_{\mathrm{AC}}\right)$ with a variable frequency $(f)$ is combined with a constant bias voltage $\left(V_{\mathrm{DC}}\right)$ which defines the working point of the measurement. Hence, the applied voltage in IS leads to a constant current through the device superimposed by a small alternating current with a phase shift $\phi$ to the applied voltage. Therewith, a direct measure of the actual device capacitance is possible. For a detailed theoretical description of IS for organic semiconductor devices, it is referred to, e.g., Refs. 8-10 and 18-20. There exist several different measurement approaches for IS. However, only two 
different procedures, the $C$ - $V$ and the $C-f$ sweeps, have been used in this article. Here, the capacitance of the device is measured while the frequency of the alternating voltage is fixed during a voltage sweep, or the voltage is fixed during a frequency sweep for the $C-V$ and the $C-f$ mode, respectively.

Impedance spectroscopy measurements have been performed with an impedance analyzer (Solartron Impedance/ Gain-Phase Analyzer SI 1260) combined with a dielectric interface (Solartron 1296). The range of available frequencies is between $10^{-5}$ and $10^{7} \mathrm{~Hz}$ and the practical voltage range for this setup between -3 and $5 \mathrm{~V}$, while the amplitude of the alternating voltage was set to $0.1 \mathrm{~V}$ r.m.s. for all measurements presented in this article.

The geometrical capacitance $\left(C_{\text {geo }}\right)$ represents the total thickness of all insulating layers for reverse bias conditions:

$$
C_{\mathrm{geo}}=\frac{\epsilon_{0} \epsilon_{r} A}{d} .
$$

Therein, $\epsilon_{0}$ and $\epsilon_{r}$ stand for the permittivity of free space and the relative permittivity of the organic layers, respectively, $A$ stands for the area of the active pixel and $d$ is the total thickness of the organic layers. Defining the geometrical capacitance in the mentioned way is possible for multilayered devices as the used organic layers exhibit very similar relative permittivities. ${ }^{19}$ The mathematical calculation of the expression is left to the reader. Note that highly conductive layers inside OLED stacks, such as doped transport layers, do not contribute to the geometrical capacitance.

\section{Displacement current measurements}

Displacement current measurements are, in contrast to IS which is a quasi-static analysis, a transient electrical method to detect the capacitance of a multilayer device such as an OLED. ${ }^{21,22}$ However, DCM additionally allows for an investigation of trapped charges. To perform DCM, a triangular voltage with variable heights and sweep rates (frequencies) is applied to the device and the current flow through the device is detected. One DCM cycle includes two sweeps with positive and negative sweep rate for the forward and the backward scan, respectively. Hence, one can probe charge carrier injection into and extraction out of the device during one measurement cycle. If the capacitance of a multi-layered device is changing during the voltage sweep due to, e.g., charge carrier injection into one particular layer species, it is even possible to calculate the amount of injected charges into that layer(s) by integrating the DCM signal ${ }^{22}$

$$
\sigma_{\mathrm{int}}(V)=\int_{V_{\mathrm{inj}}}^{V}\left(C_{\mathrm{dev}}-C_{\mathrm{geo}}\right) \frac{C_{1}+C_{2}}{C_{2}} d V^{\prime},
$$

where $\sigma_{\text {int }}(V)$ represents the injected charge carrier density during the forward direction of the DCM. In this formula, $C_{1}, C_{2}$, and $C_{\mathrm{dev}}$ stand for the hole transport layers, the electron transport layers, and the actual device capacitance, respectively, and $V_{\mathrm{inj}}$ is the injection voltage at which one particular layer of the multi-layer device (in our case the hole transport layers) becomes conductive. The analogous method can be used for calculating the amount of extracted charge carriers during the backward sweep of the DCM curve. Furthermore, it is possible to detect changes during a first and a second DCM cycle accounting for, e.g., trapped charge carriers. Thus, DCM is a powerful tool to analyze degradation induced changes of the electrical properties of OLEDs. ${ }^{23}$

All DCM investigations presented in this article have been performed at room temperature with appropriate slow sweep rates $(\leq 100 \mathrm{~V} / \mathrm{s})$ using an FCE-1 measurement system for ferroelectrics (Toyo Corp.).

\section{THEORY}

In order to analyze the drop in luminance during the electrical degradation, one has first to define the measured quantity. The external quantum efficiency (EQE) of organic light-emitting diodes is given by four independent factors: ${ }^{24}$

$$
\eta_{\text {ext }}=\gamma \cdot \eta_{\mathrm{r}} \cdot q_{\text {eff }}(q) \cdot \eta_{\text {out }} \equiv \eta_{\text {int }} \cdot \eta_{\text {out }} \cdot
$$

Therein, $\gamma$ represents the charge carrier balance. $\eta_{\mathrm{r}}$ accounts for the fraction of created excitons which are quantum-mechanically allowed to decay under creation of a photon. Thus, $\eta_{\mathrm{r}}$ becomes unity for phosphorescent emitters ${ }^{25-27}$ and can clearly exceed the spin-statistical limit of 25\% (Refs. 28-36) in the fluorescent case. The third factor stands for the effective radiative quantum efficiency of the emitting system and depends on its intrinsic value $q$, which is modified by the Purcell effect taking into account the microcavity-like structure. ${ }^{37-39}$ The last factor gives the outcoupling efficiency of the device and takes into account that not all photons created inside the OLED can contribute to light-emission to the outside world. This factor is strongly influenced by the refractive indices and thicknesses of all used organic and inorganic layers and the orientation of the transition dipole moments of the emitting species. ${ }^{35,39-43}$ The first three factors can be combined to the so-called internal quantum efficiency $\eta_{\text {int }}$.

With this information, it is now possible to investigate the changes of all four factors determining the EQE during electrical aging in order to get a comprehensive insight in the underlying mechanisms and possible future opportunities for improvement of long-term stability during electrical operation. Possible impacts on each of the four factors are

(1) Decreasing charge carrier balance due to increasing charge carrier injection barriers, modified charge transport properties in the different layers and/or nonradiative recombination via trap states.

(2) Decreasing effective radiative quantum efficiency as a result of changes of the position of the recombination zone in the EML (Purcell factor) and an increased nonradiative rate (e.g., generation of deactivated emitting molecules or triplet-polaron-quenching by trapped charges).

(3) Decreasing outcoupling factor due to a shift of the position of the emission zone and reorientation of the transition dipole moments of the emitting species. 
As mentioned in the list above, one possible explanation for a decreasing radiative quantum efficiency of the emitting system during degradation can be an enhanced non-radiative rate due to a TPQ process with trapped charges. In order to verify this assumption, one needs a measure for the nonradiative rate, which is obtainable via TRELS, and an expression for the TPQ induced changes of the excited states lifetime. Therefore, we modify the approach described by Reineke et al ${ }^{44}$ for free charge carriers:

The changes of the exciton population $n_{e x}$ in the EML during current flow are given by

$$
\frac{d n_{e x}}{d t}=-\frac{n_{e x}}{\tau_{0}}-\Gamma_{T P Q} \cdot p_{c}(j) \cdot n_{e x}+G .
$$

Therein, $\tau_{0}$ is the exciton lifetime in the OLED cavity without current flow according to Eq. (2), $G$ is a constant term describing exciton generation under steady-state operation, $\Gamma_{T P Q}$ is the triplet-polaron-quenching rate, and $p_{c}(j)$ is the charge carrier density through the device.

For the excited states lifetime in a TRELS experiment, the factor $G$ becomes zero due to vanishing current flow after the excitation pulse. $p_{c}(j)$ is replaced by the trap density $\left(n_{\text {trap }}\right)$ assumed, for simplicity, to be homogeneous across the emission zone.

Hence, the above given equation changes to

$$
\begin{gathered}
\frac{d n_{e x}}{d t}=-\frac{n_{e x}}{\tau_{0}}-\Gamma_{T P Q} \cdot n_{\text {trap }} \cdot n_{e x} \\
\Rightarrow \frac{d n_{e x}}{d t}=-n_{e x}\left(\frac{1}{\tau_{0}}+\Gamma_{T P Q} \cdot n_{t r a p}\right) .
\end{gathered}
$$

Now, the total exciton decay rate $\Gamma_{t o t}$ is given by the expression in parentheses on the right-hand side of the equation which can be re-written as

$$
\Gamma_{t o t}=F \cdot \Gamma_{r}+\Gamma_{n r}+\Gamma_{T P Q} \cdot n_{\text {trap }},
$$

where the identity for $\tau_{0}$ (Eq. (2)) has been used.

With this expression and the assumption of vanishing $n_{\text {trap }}$ in the pristine device and unaffected radiative rate (during the degradation process), the normalized excited states lifetime is given by

$$
\begin{gathered}
\frac{\tau}{\tau_{0}}=\frac{\Gamma_{t o t, 0}}{\Gamma_{t o t}}=\frac{F \cdot \Gamma_{r, 0}+\Gamma_{n r, 0}}{F \cdot \Gamma_{r, 0}+\Gamma_{n r, 0}+\Gamma_{T P Q} \cdot n_{\text {trap }}} \\
=\frac{\frac{1}{\tau_{0}}}{\frac{1}{\tau_{0}}+\Gamma_{T P Q} \cdot n_{\text {trap }}}=\frac{1}{1+\tau_{0} \cdot \Gamma_{T P Q} \cdot n_{\text {trap }}} \\
\Rightarrow \tau=\frac{\tau_{0}}{1+\tau_{0} \cdot \Gamma_{T P Q} \cdot n_{\text {trap }}} .
\end{gathered}
$$

Therewith, we obtain an expression, which can be used to analyze degradation induced changes of the exciton lifetime as a function of the trap density in the EML and the TPQ quenching rate.

In addition to quenching excitons, traps can also affect the external quantum efficiency of OLEDs in a second way, namely, by causing non-radiative recombination of charge carriers (trap-assisted recombination). ${ }^{45-50}$ Although the number of injected electrons and holes does not necessarily change, this will effectively lead to a reduction of the charge balance factor $\gamma$, because part of the carriers does no longer form excitons. Using the definition of $\gamma=j_{r e c} / j_{t o t}$ and considering that the (radiative) recombination current $\left(j_{\text {rec }}\right)$, i.e., the fraction leading to excitons, is given by the total current $\left(j_{t o t}\right)$ reduced by the non-radiative recombination current $\left(j_{n r}\right)$, it follows that $\gamma=\frac{j_{t o t}-j_{n r}}{j_{t o t}}=1-\frac{j_{n r}}{j_{t o t}}$.

From the continuity equation $\frac{1}{e} \frac{\partial j}{\partial x}=R_{\mathrm{BMR}}+R_{\mathrm{TAR}}$ with $R_{\mathrm{BMR}}=2 \frac{e}{\epsilon} \mu n^{2} \quad$ (bimolecular recombination rate) and $R_{\mathrm{TAR}}=\frac{e}{\epsilon} \mu n_{\text {trap }} n$ (trap-assisted recombination rate), following the simplified treatment by Coehoorn et al. ${ }^{51}$ and Kuik et $a l .{ }^{49}$ and neglecting the variation of the current over the width $d$ of the recombination zone (emission layer), one obtains the following expressions for both currents:

$$
j_{t o t}=2 \frac{e^{2}}{\epsilon} \mu d n^{2} \quad \text { and } \quad j_{n r}=\frac{e^{2}}{\epsilon} \mu n_{\text {trap }} d n .
$$

Therein, equal mobilities $(\mu)$ as well as carrier densities of electrons and holes $(n)$ are assumed for the bimolecular case and, overall, bimolecular recombination is assumed to be dominant as compared to trap-assisted recombination. The latter will certainly be no longer fulfilled for very large trap densities; but then $\gamma \rightarrow 0$. From these equations, one finally obtains

$$
\gamma=1-\frac{n_{\text {trap }}}{2 n}
$$

with $n$ depending on the applied current.

Furthermore, since the $\mathrm{EQE}$ is directly proportional to the luminance $(L)$, the relative changes of $L$ with aging time can be written as

$$
\frac{L}{L_{0}}=\frac{\gamma \cdot \eta_{\mathrm{r}} \cdot q_{\mathrm{eff}} \cdot \eta_{\text {out }}}{\gamma_{0} \cdot \eta_{\mathrm{r}, 0} \cdot q_{\mathrm{eff}, 0} \cdot \eta_{\mathrm{out}, 0}} .
$$

Accounting for cavity effects leads to the effective radiative quantum efficiency using the Purcell factor $F$ :

$$
q_{\mathrm{eff}}=\frac{F \cdot \Gamma_{r}}{F \cdot \Gamma_{r}+\Gamma_{n r}} .
$$

If only the changes of the non-radiative rate of the emitting system, e.g., due to TPQ at trapped charges, and the charge carrier balance are responsible for the degradation process while outcoupling factor, radiative exciton fraction and radiative rate of the emitter remain unaffected, Eq. (14) is reduced to

$$
\begin{aligned}
\frac{L}{L_{0}} & =\frac{q_{\mathrm{eff}}}{q_{\mathrm{eff}, 0}} \cdot \frac{\gamma}{\gamma_{0}}=\frac{F \cdot \Gamma_{r} \cdot\left(F \cdot \Gamma_{r}+\Gamma_{n r, 0}\right)}{F \cdot \Gamma_{r} \cdot\left(F \cdot \Gamma_{r}+\Gamma_{n r}\right)} \cdot \frac{\gamma}{\gamma_{0}} \\
& =\frac{F \cdot \Gamma_{r}+\Gamma_{n r, 0}}{F \cdot \Gamma_{r}+\Gamma_{n r}} \cdot \frac{\gamma}{\gamma_{0}}=\frac{\tau}{\tau_{0}} \cdot \frac{\gamma}{\gamma_{0}} .
\end{aligned}
$$

With the assumption that the trapped charges created during degradation are responsible for the loss of radiative 


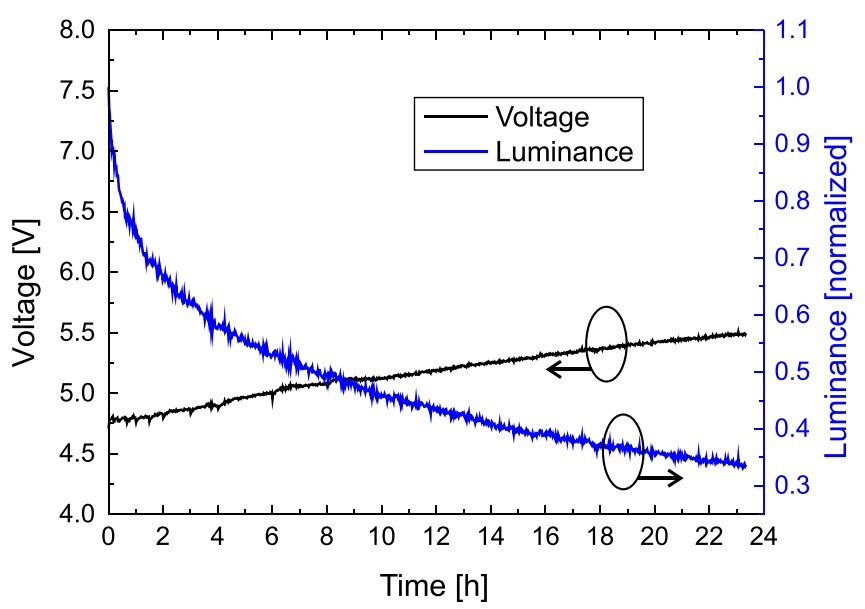

FIG. 2. Degradation behavior of the OLED under investigation under constant current conditions $\left(1 \mathrm{~mA} / \mathrm{cm}^{2}\right)$ resulting in an initial luminance value of around $500 \mathrm{~cd} / \mathrm{m}^{2}$.

quantum efficiency and charge carrier balance due to TPQ and TAR, respectively, Eq. (16) can be expressed as a function of the trap density by using Eqs. (10) and (13) as follows:

$$
\frac{L}{L_{0}}=\frac{q_{\mathrm{eff}}}{q_{\mathrm{eff}, 0}} \frac{\gamma}{\gamma_{0}}=\frac{1}{1+\tau_{0} \cdot \Gamma_{T P Q} \cdot n_{\text {trap }}} \cdot\left(1-\frac{n_{\text {trap }}}{2 n}\right) .
$$

\section{EXPERIMENTAL RESULTS}

An accelerated aging protocol under constant current conditions $\left(1 \mathrm{~mA} / \mathrm{cm}^{2}\right)$ was performed with the OLED displayed in Fig. 1 resulting in an initial luminance of about $500 \mathrm{~cd} / \mathrm{m}^{2}$ at $4.8 \mathrm{~V}$. Figure 2 shows the decrease of the luminance and the simultaneous increase of the required voltage. The drop in the luminance follows a stretched exponential decay, which is an indication for the creation of nonradiative recombination centers inside the EML. ${ }^{51,52}$ After 15 hours of degradation, the luminance is only $40 \%$ of the initial value and the required voltage increases by $0.5 \mathrm{~V}$ to $5.3 \mathrm{~V}$.

The current density-voltage-luminance characteristics (IVL) of the device during aging are shown in Figure 3. While the I-V-curve shows only little changes during the electrical aging, a strong decrease of the luminance is clearly visible. However, the onset of the luminance and of the current does not change and remains constant at about $2.4 \mathrm{~V}$ over the whole degradation time.

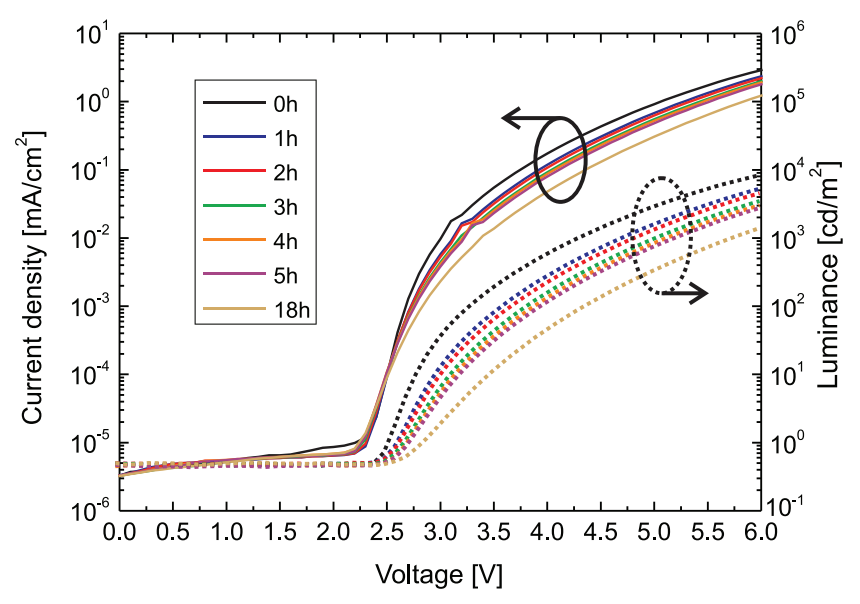

FIG. 3. Current density-voltage (solid lines) and luminance-voltage (dotted lines) characteristics during the degradation process. The onset voltage in both cases remains constant over the whole aging time.

To analyze the decrease in luminance in more detail, TRELS has been performed during the degradation process. It should be noted that no additional degradation induced by performing TRELS was visible. However, the excited states lifetime recorded after a short electrical pulse with a height of $6 \mathrm{~V}$ and a duration of $25 \mu$ s was subsequently decreased from an initial value of $0.81 \mu$ s to $0.61 \mu$ s after $18 \mathrm{~h}$ electrical aging, while the emission spectrum did not change during this time (Fig. 4). Hence, significant changes of the recombination/emission zone during aging are very unlikely, which in turn implies that the outcoupling factor of the device remains constant and can be excluded as one possible degradation mechanism decreasing the luminance. Furthermore, the changes of the excited states lifetime can be explained by creation of quenching centers increasing the non-radiative decay rate of the excited dye molecules, while the radiative rate is assumed to stay constant for the used emitting system. Note that due to the moderate negative electrical bias during the off state residual free charges are extracted in order to minimize their influence during the decay of the EL signal. ${ }^{16}$ However, even for different excitation conditions (with and without negative bias during the off state and varying positive pulse height), the same values of the decay time were obtained. The long-living part of the measurements shown in Fig. 4 is a consequence of bad signal to noise ratio due to the very small pixel size of $1 \mathrm{~mm}^{2}$. Thus, the fits were only performed in the clear monoexponential region.
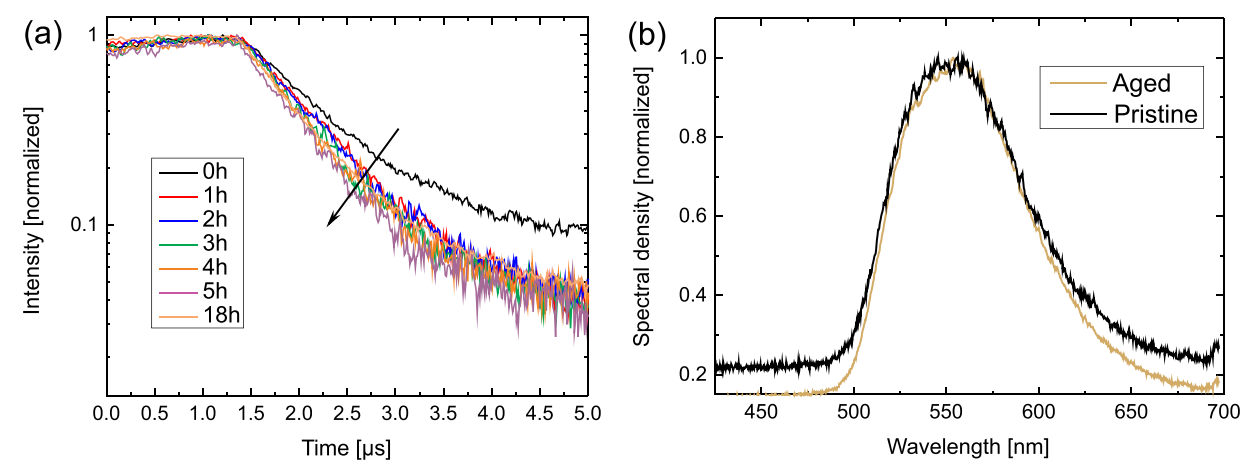

FIG. 4. (a) Excited states lifetime investigations via TRELS during the degradation process. The excited states lifetime (mono-exponential fits) is subsequently decreased with aging time. (b) Emission spectra of the pristine device and after $18 \mathrm{~h}$ electrical stressing. 


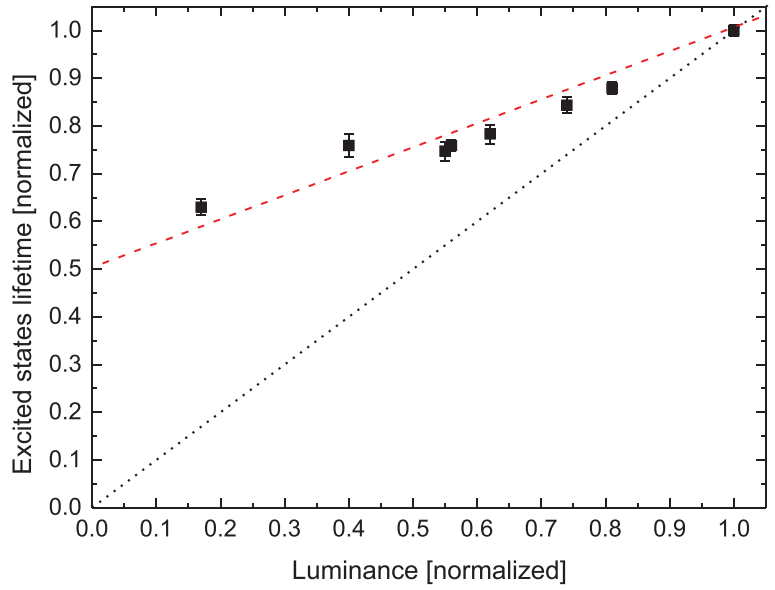

FIG. 5. Excited states lifetime (dots) normalized by the pristine value of $0.81 \mu \mathrm{s}$ as a function of the normalized luminance during degradation. The data can be fitted with a linear function (red dashed line) with a slope of 0.5 . The one-to-one correlation is shown as black dotted line.

Analyzing the measured TRELS data in Figure 4 in more detail results in a linear correlation between the decrease of the normalized excited states lifetime and the luminance with a slope of about 0.5 as shown in Fig. 5. This behavior indicates that the changes of the excited states lifetime and therewith of the effective radiative quantum efficiency of the used emitting system can only explain part of the drop in luminance during electrical aging as expected from Eq. (16), if an unmodified outcoupling factor, charge carrier balance, radiative decay rate, and radiative exciton fraction are assumed (one-to-one correlation). Actually, the loss in radiative quantum efficiency of the emitting system can only account for $50 \%$ of the degradation induced decrease of the luminance and other effects lowering the EQE, such as changes of the charge carrier balance of the device, have to be taken into account. Therefore, a comprehensive analysis of the degradation induced modifications of the electrical properties has been performed using IS and DCM.

The impedance response of the pristine device is shown in Fig. 6. The geometrical capacitance evaluated from both measurement types, $C-V$ and $C-f$, confirms the layer thicknesses of all organic layers mentioned in Fig. 1 and can be described by three individual $R C$-elements for the HTL, the EML and the ETL, respectively. The capacitance of the HIL (HATCN) does not contribute to $C_{\text {geo }}$ due to its high conductivity.

Interestingly, the actual capacitance of the device increases already for slightly negative voltages in the $C-V$ sweep due to hole injection into the HTL and the EML of the device and subsequent hole accumulation at the EML/ETL interface. This is caused by an interface charge, generated at the EML/ETL interface by intrinsic spontaneous orientation polarization of the BPhen layer. This phenomenon was already observed for a manifold of polar electron transporting materials ${ }^{18,53-55}$ such as $\mathrm{BCP}^{21}$ (2,9-dimethyl-4,7diphenyl-1,10-phenanthroline) which is very similar to the used BPhen molecule in this study. Furthermore, in the $C-f$ response for voltages over $1.5 \mathrm{~V}$, two plateaus are visible corresponding to the hole accumulation regimes of the HTL and the EML for higher and lower relaxation frequencies (frequency at which the increasing capacitance reaches half of the next plateau height), respectively. Hence, it is possible to distinguish between these two layers in the $C$ - $f$ sweeps so that electrical changes during degradation can be unambiguously assigned to the corresponding layers.
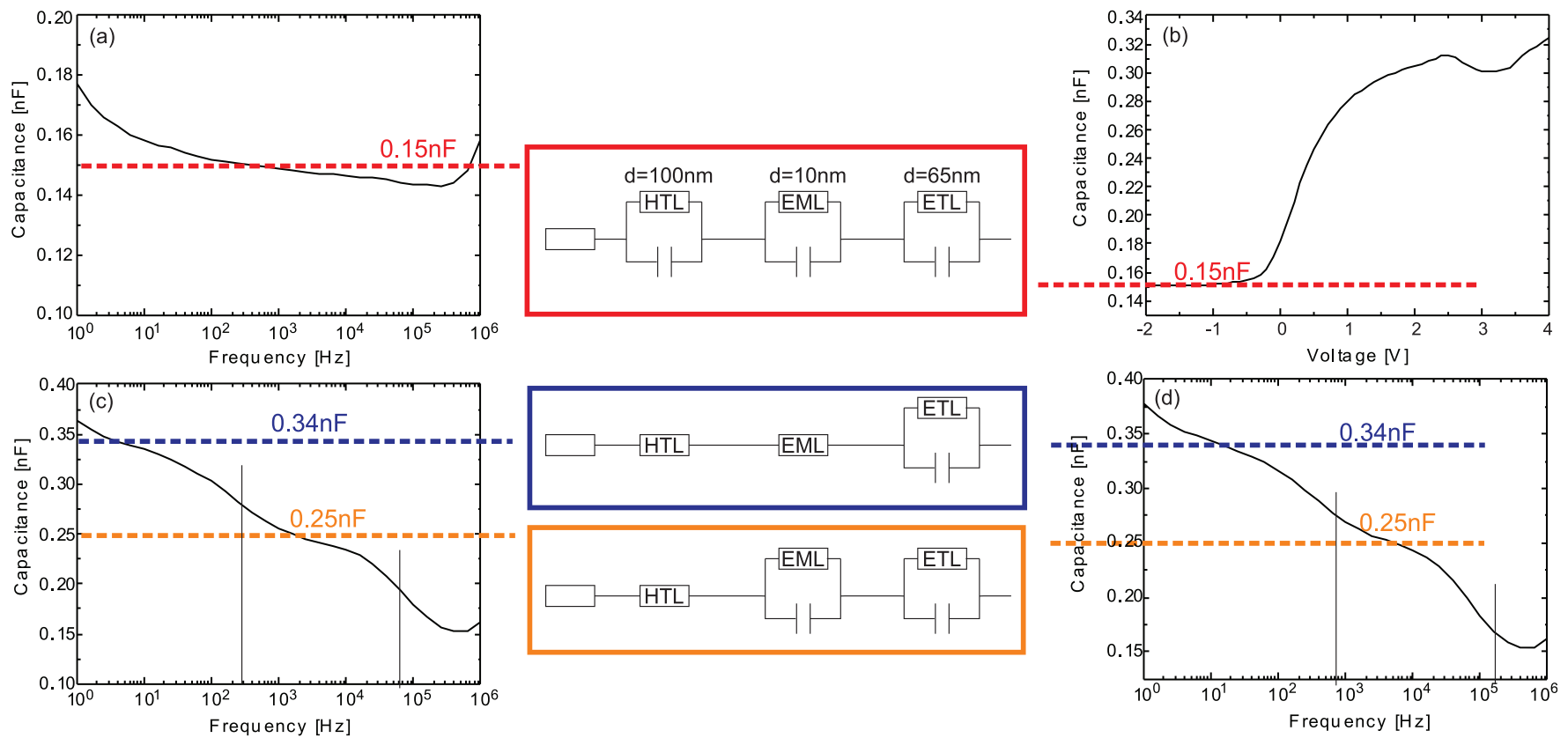

FIG. 6. (a)-(d) Impedance measurement results and equivalent circuit models (middle column) for the detected capacitance. (a) $C$ - $f$ measurement at reverse bias conditions of $-1 \mathrm{~V}$. (b) $C$ - $V$ data detected at a frequency of $133 \mathrm{~Hz}$. Both measurements reproduce the geometrical capacitance of the three layer system (neglecting the high conductive HATCN layer) in a proper way. Hole injection for the pristine device takes place at around $-0.5 \mathrm{~V}$. (c) and (d) represent $C-f$ measurements at $1.5 \mathrm{~V}$ and $2.4 \mathrm{~V}$, respectively, clearly showing a two step behavior for voltages where hole accumulation takes place. The two plateaus correspond to the conductivity regimes of the HTL and the EML for higher and lower relaxation frequencies, respectively. The vertical lines are indicating the position of the relaxation frequencies of the two detectable layers. 

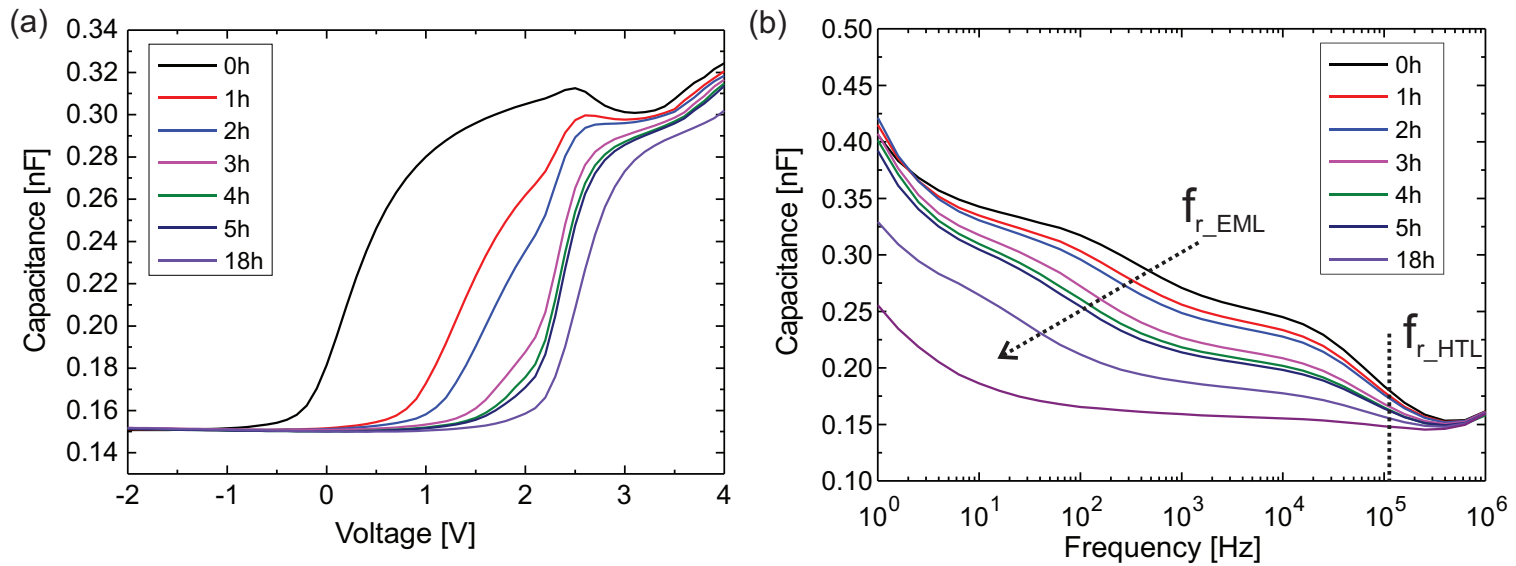

FIG. 7. Impedance measurement results for different degradation steps. (a) The hole injection voltage is shifting to higher voltages with aging time in the $C$ - $V$ sweeps for a fixed frequency of $133 \mathrm{~Hz}$ indicating a compensation or changes of the interface charge. The capacitance for hole accumulation is not changing. (b) The relaxation frequencies (measure for the conductivity of a layer) in the $C$ - $f$ sweeps (at $2.5 \mathrm{~V}$ DC bias) for the two detectable layers show different influences of the degradation process. While the conductivity of the HTL is not changing, the relaxation frequency of the EML shifts to lower frequencies and hence the conductivity decreases with aging time.

The IS measurements during electrical aging are presented in Fig. 7. In the $C$ - $V$ sweeps (for a fixed frequency of $133 \mathrm{~Hz}$ ), it is clearly visible that the hole injection voltage is shifting to higher voltages, corresponding to a compensation of the interface charge induced by the ETL due to the creation of positively charged trap states in the HTL and/or EML or due to reorganization of the orientation polarization in the BPhen layer caused by the charge transport through it. Furthermore, the capacitance for hole accumulation for higher voltages than injection remains constant indicating that the position of the hole accumulation interface is not changing due to electrical aging. The $C-f$ sweeps at a fixed DC bias of $2.5 \mathrm{~V}$ indicate changes of the hole conductivity of the EML during the degradation process. Interestingly, the relaxation frequency (measure of the conductivity of certain layers in the device) for the HTL remains constant at a relatively high value of about $10^{5} \mathrm{~Hz}$ during electrical aging, while the relaxation frequency of the EML is clearly shifted to lower values representing a decrease in its conductivity. A possible explanation for this behavior, which is consistent with the results of the $C-V$ sweeps, is the creation of positively charged trap states in the EML. However, a clear verification is not possible with IS. Hence, additional displacement current measurements have been performed during electrical aging.

With DCM, it is possible to analyze the density of trapped charges in the device by simply comparing the injected charge density directly after a detrapping process and a subsequently following DCM sweep. For the detrapping process we used white light from an incandescent electric lamp being absorbed from the device during reverse bias conditions of $-2 \mathrm{~V}$ for $20 \mathrm{~s}$ to ensure release and extraction of captured charge carriers. Figure 8 displays two exemplary DCM responses for the pristine device and after $2 \mathrm{~h}$ degradation for a sweep rate of $100 \mathrm{~V} / \mathrm{s}$. While in the pristine case the subsequent DCM curves are almost identical, a difference in the forward scan between the first and the second DCM cycle is clearly detectable for the aged device. As mentioned before, integrating the current flow in a DCM investigation between $V_{\text {th }}$ and $V_{\text {inj }}$ equals the charge carrier density injected into the device. Hence, the difference between the first and the second DCM cycle corresponds directly to the trapped charge density (see Eq. (4)). Note that there is a shift of $V_{\text {inj }}$ from 0.25 to $0.60 \mathrm{~V}$ between both sweeps, while $V_{\text {th }}$ remains unaffected. This observation (creation of trap states) is in good agreement with the changes in the hole injection voltage detected via IS. Additionally, it is worth mentioning that the curve shape of the backward scan of the DCM curves is totally identical for the first and the second sweep cycle for the pristine as well as the aged device. This reveals that the conductivity of the hole transport layer is not changing and the created traps, which strongly influence the hole mobility, are located inside the EML, e.g., near the EML/ETL interface. This is, again, in excellent

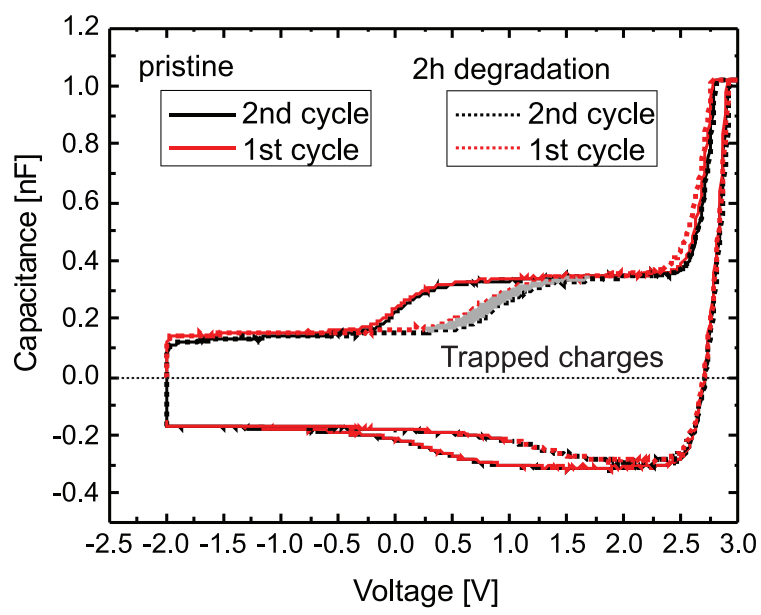

FIG. 8. 1st and 2nd DCM (100 V/s) cycle after detrapping via white light exposure under reverse bias conditions for the pristine state and after $2 \mathrm{~h}$ degradation time. The differences between the first and the second measurement cycle in the injected charge carriers (forward direction) correlates with the trapped charge density (see Eq. (4)). While there are no trapped charges in the pristine case, a reasonable amount of injected charge carriers is trapped after $2 \mathrm{~h}$ aging time (indicated as gray area). The backward scan response of the DCM curves is not changing for the different DCM cycles. This gives evidence for locating of the trap states inside the EML of the device. 


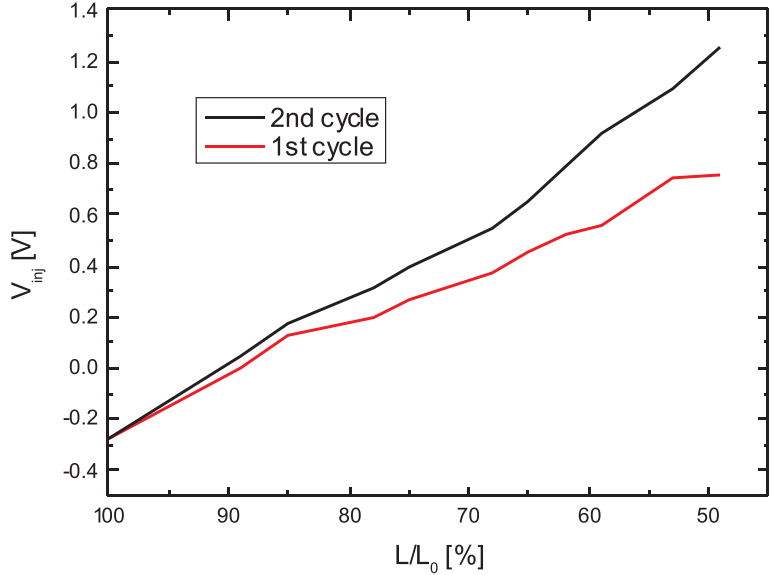

FIG. 9. Hole injection voltage as a function of the decreasing luminance during electrical degradation for the first and the second DCM $(100 \mathrm{~V} / \mathrm{s})$ cycle after detrapping by light absorption under reverse bias conditions. The differences result from the creation of trap states in the EML. The hole injection voltage for the first cycle cannot be fully recovered by the detrapping process.

agreement with the results achieved by IS. If the hole conductivity of the corresponding transport layer, here the HTL of the device, were changed due to the creation of trap states, the charge carrier extraction trough this layer would be influenced and the voltage onset of hole extraction would shift to lower or even negative voltages. This behavior was proven by targeted introduction of trap states into transport layers resulting in the mentioned behavior. However, because the detailed explanation of this effect is far beyond the focus of this investigation, and the DCM results are in excellent agreement with the IS findings (no changes of the hole conductivity of the HTL but strong changes of the EML), the corresponding measurements are not shown here.

Nevertheless, the creation of rechargeable trap states alone cannot explain all changes of the electrical behavior during degradation, because it is not possible to achieve a full recovery (of the hole injection voltage) by the detrapping process (Fig. 9). Hence, a second effect that is influencing the DCM curves has to be present in the device. One possible additional process is the creation of deep trap states which cannot be released by the mentioned detrapping process or, what is more likely, the orientation polarization of the ETL is changed by electrical transport.

So far, we have not presented evidence that the created trap states are really filled with injected holes. Therefore, Fig. 10 shows the first and the second DCM cycle after detrapping for voltage sweeps between $-2 \mathrm{~V}$ and $3 \mathrm{~V}$ (where already electron injection takes place) as well as between $-2 \mathrm{~V}$ and $2 \mathrm{~V}$ (hole accumulation at the EML/ETL interface; no electrons are injected into the device) for different degradation steps. It is clearly visible that the behavior for both sweep cycles does not depend on the highest applied voltage and, hence, the filling of the trap states is identical if only holes are injected into the device as well as if bipolar charge carrier injection is present during the DCM cycle. This gives evidence that the trap states are filled with injected holes and electrons only play a minor role for this phenomenon.

Moreover, it is now possible to correlate the degradation induced creation of positively charged trap states extracted from DCM with the decrease in excited states lifetimes. Kondakov et al. $^{45}$ analyzed the degradation process of the used CBP host in detail and found that excitation, e.g., by electrical operation, leads to cleavage of exocyclic $\mathrm{C}-\mathrm{N}$ bonds in CBP and subsequent creation of neutral radicals and their reduced/oxidized forms, acting as hole traps. For the calculation of the trapped charge density (from the DCM curves), we assumed a constant emission profile and hence a constant trap concentration over $2 \mathrm{~nm}$ of the EML directly at the interface to the ETL as IS and DCM analysis (hole accumulation) reveal an emission zone close to the EML/ETL interface.

As mentioned in the theory section, we use a modified triplet-polaron-quenching model for trapped charges as we have (a)

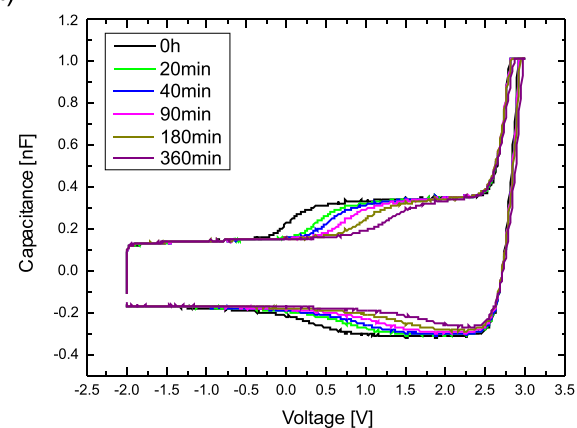

(c)

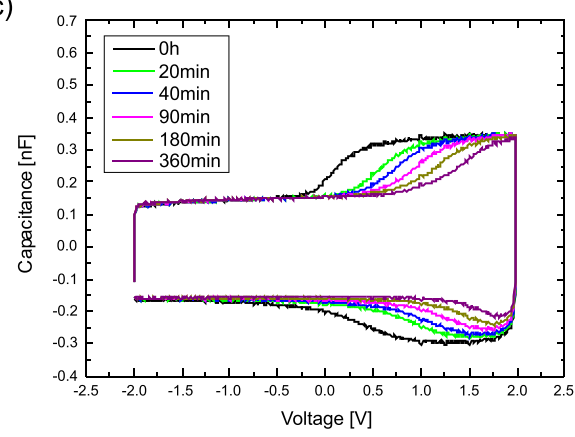

(b)

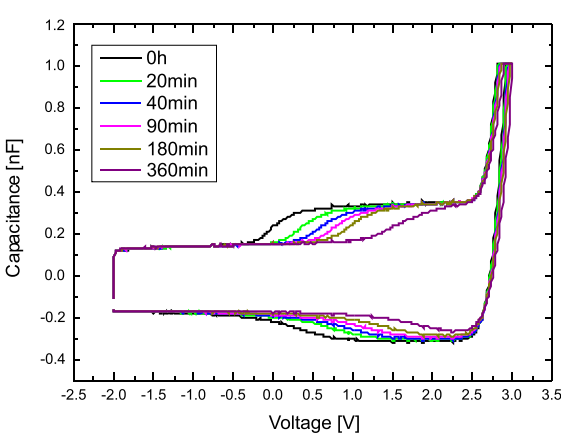

(d)

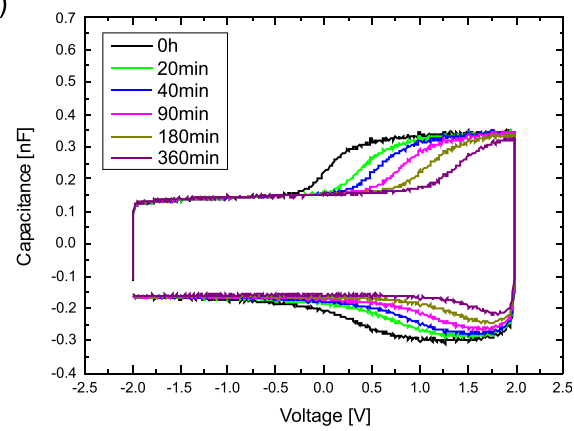

FIG. 10. DCM (100 V/s) responses for different degradation steps. (a) and (b) show the measurements in a voltage range of $-2 \mathrm{~V}$ to $3 \mathrm{~V}$ of the first and the second sweep cycle, respectively. (c) and (d) show the measurements in a voltage range of $-2 \mathrm{~V}$ to $2 \mathrm{~V}$ of the first and the second sweep cycle, respectively. Due to the identical behavior of the measurements for both voltage ranges, one can conclude that the trap states are positively charged due to hole injection only. 
found them to originate from the electrical aging process of the host of the EML. Using Eq. (11) for the changes of the excited states lifetimes determined via TRELS and the trapped charge density from DCM, one can fit the measured data in a reasonable way with the TPQ rate as remaining fit parameter. Figure 11 shows the measured data together with the mentioned fit using Eq. (11). The fit reproduces the data set very well resulting in a TPQ rate of $(0.23 \pm 0.03) \times 10^{-13} \mathrm{~cm}^{3} / \mathrm{s}$ which is in excellent agreement with published results. ${ }^{44,56}$ Hence, we are confident that the mechanism for the decrease of the effective radiative quantum efficiency of the emitting system during degradation is based on a TPQ process and is therewith directly correlated with the creation of trap states in the EML and the EL-lifetime is only very little influenced by residual free charges. However, according to the results from Figure 5, only about $50 \%$ of the luminance loss during degradation originates from the TPQ mechanisms with trapped charges.

Additionally, as already mentioned before, the creation of trap states is influencing the charge carrier balance in a non-negligible way. Not only does hole injection into the EML start at higher voltages and the hole transport dynamics change in the EML, but also will trap states cause trapassisted non-radiative recombination, ${ }^{45-50}$ which in turn can reduce the charge carrier balance of the device.

To prove this assumption, we use Eq. (17) to fit the drop in luminance as a function of the trap density. With the TPQ rate achieved from fitting the excited states lifetimes as a function of the trap density (Fig. 11), the only remaining fit parameter in Eq. (17) is the charge carrier density due to the constant current flow through the device. Figure 12 shows the decrease in luminance as a function of the measured trap density together with the fit using Eq. (17) as well as the individual contributions of TPQ and TAR. The fit describes the data in a reasonable way, however, the extracted charge carrier density seems to be overestimated $\left(n \approx 10^{19}\right.$ at the applied current density). This might be caused by the simplified assumptions regarding the description of the charge carrier balance (equal mobilities and carrier densities of

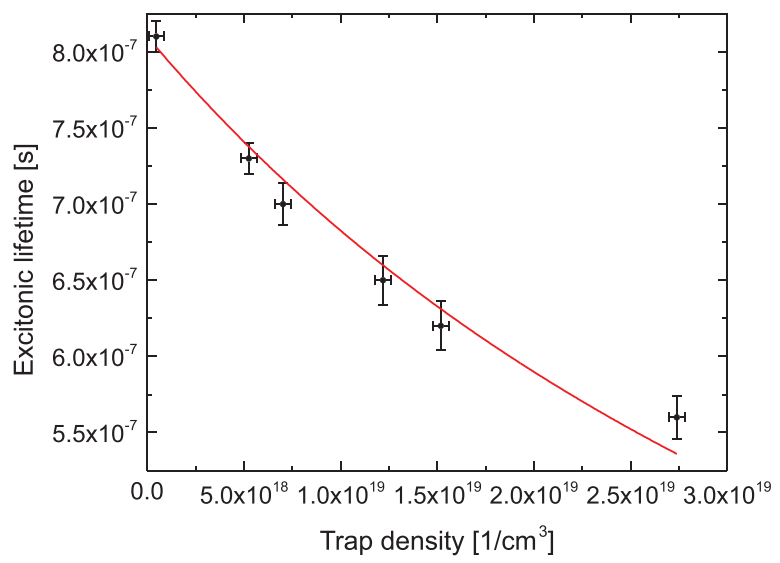

FIG. 11. Dots: Excited states lifetime from TRELS experiments as a function of the created trapped charge carrier density determined via DCM investigations during electrical degradation. Solid line: Fit of the experimental data using the modified Triplet-Polaron-Quenching model (Eq. (11)). The fit describes the measured data in an excellent way and results in a reasonable value of the TPQ rate of $(0.23 \pm 0.03) \times 10^{-13} \mathrm{~cm}^{3} / \mathrm{s}$.

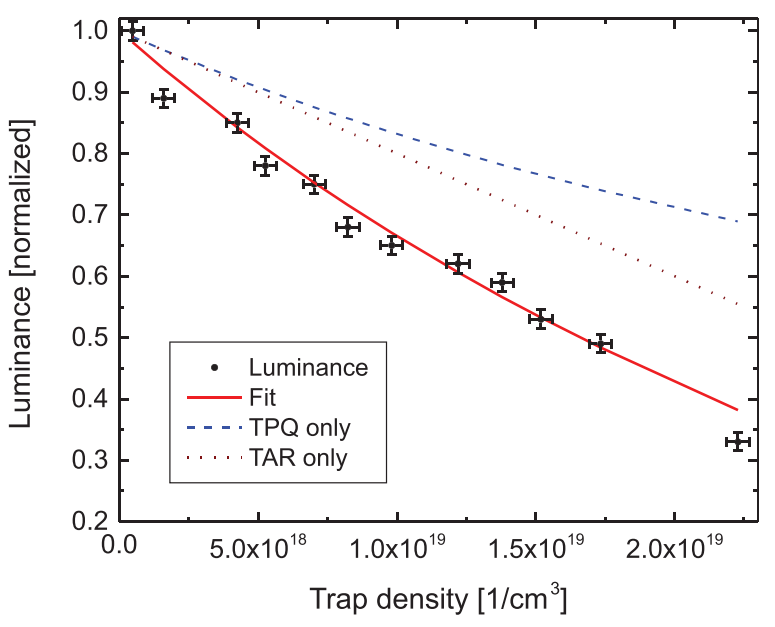

FIG. 12. Normalized luminance as a function of trap density (dots). The red line represents the fit including the individual contributions of triplet-polaron-quenching (blue dashed line) and trap-assisted non-radiative recombination (brown dotted line), respectively.

electrons and holes as well as bimolecular recombination has been assumed to be dominant compared to TAR).

However, although the microscopic mechanism behind the loss in charge carrier balance is not analyzed in detail here, it is conceivable that one half of the luminance loss during electrical aging is caused by this process.

\section{CONCLUSION}

In summary, we analyzed the degradation behavior of organic light-emitting diodes comprising the well known phosphorescent $\operatorname{Ir}(\text { ppy })_{3}$ molecule doped into a CBP matrix as emitting system. We investigated both the optical and the electrical changes during an accelerated electrical aging protocol via TRELS as well as DCM and IS, respectively. We identified two main mechanisms for the luminance loss during degradation: decreasing charge carrier balance as well as reduced effective radiative quantum efficiency of the emitting system, both induced by the formation of trap states in the EML. The charge carrier balance is probably deteriorated by a reorientation effect of the spontaneous orientation polarization of the electron transporting layer BPhen affecting the interface charge at the EML/ETL interface or, as fitting the results with a simplified model reveals, more likely by trap-assisted non-radiative recombination of the charge carriers. At the same time, the created trap states act also as quenching centers for the phosphorescent decay of the Ir(ppy $)_{3}$ molecules. We could describe the observed behavior of the excited states lifetime with a modified TPQ model for trapped charges that confirms the direct correlation between the created trap density and the loss in radiative quantum efficiency. Together with trap-assisted non-radiative recombination, it was possible to explain the loss in external quantum efficiency of the device with $50 \%$ contribution of each of both mechanisms, and therewith the degradation process was analyzed in a comprehensive way. As there exists evidence that the matrix molecules CBP are unstable due to electrical excitation, we ascribe the formation of the positively charged trap states exclusively to the matrix material. 
Hence, the choice of both, the electron transporting material, which often exhibits spontaneous orientation polarization and the matrix material of the emitting system is of major importance to improve OLED performance in terms of long term stability under electrical operation.

\section{ACKNOWLEDGMENTS}

We acknowledge financial support by the German Federal Ministry of Education and Research (BMBF) under the Contract No. 13N12240 "OLYMP" and by the Deutsche Forschungsgemeinschaft (Contract No. BR 1728/13-1). Tobias D. Schmidt also acknowledges support by the Japanese Society for the Promotion of Science (JSPS Summer Program 2014).

${ }^{1}$ R. Cheng, Wall Street J., September 6th (2010), available at http:// www.wsj.com/articles/SB10001424052748703713504575475553352680096. ${ }^{2}$ D. Gilbert, International Business Times, January 5 (2015), available at http://www.ibtimes.co.uk/ces-2015-lg-bets-big-oled-tvs-flexible-display600m-investment-1481995.

${ }^{3}$ Commercial white OLEDs are offered since 2010, e.g., by Osram and Philips.

${ }^{4}$ See http://www.oled-info.com/osram-developed-87-lmw-white-oledpanel-production-soon.

${ }^{5}$ Z. D. Popovic, H. Aziz, N.-X. Hu, A. Ioannidis, and P. N. M. dos Anjo, J. Appl. Phys. 89, 4673 (2001).

${ }^{6}$ Z. D. Popovic, H. Aziz, A. Ioannidis, N.-X. Hu, and P. N. M. dos Anjo, Synth. Met. 123, 179 (2001).

${ }^{7}$ T. D. Schmidt, D. S. Setz, M. Flämmich, B. J. Scholz, A. Jaeger, C. Diez, D. Michaelis, N. Danz, and W. Brütting, Appl. Phys. Lett. 101, 103301 (2012).

${ }^{8}$ N. C. Giebink, B. W. D'Andrade, M. S. Weaver, J. J. Brown, and S. R. Forrest, J. Appl. Phys. 105, 124514 (2009).

${ }^{9}$ D. Y. Kondakov, J. Appl. Phys. 104, 084520 (2008).

${ }^{10}$ S. Nowy, W. Ren, A. Elschner, W. Lövenich, and W. Brütting, J. Appl. Phys. 107, 054501 (2010).

${ }^{11}$ Y. Zhou, J. W. Shim, C. Fuentes-Hernandez, A. Sharma, K. A. Knauer, A. J. Giordano, S. R. Marder, and B. Kippelen, Phys. Chem. Chem. Phys. 14, 12014 (2012).

${ }^{12} \mathrm{~B}$. Bröker, "Electronic and structural properties of interfaces between electron donor \& acceptor molecules and conductive electrodes," Ph.D. dissertation (Humboldt-University, Berlin, 2010).

${ }^{13}$ D.-H. Lee, Y.-P. Liu, K.-H. Lee, H. Chae, and S. M. Cho, Org. Electron. 11, 427 (2010).

${ }^{14}$ B. D. Chin, Y. Choi, H.-I. Baek, and C. Lee, Proc. SPIE 7415, 741510 (2009).

${ }^{15}$ M. Y. Chan, C. S. Lee, S. L. Lai, M. K. Fung, F. L. Wong, H. Y. Sun, K. M. Lau, and S. T. Lee, J. Appl. Phys. 100, 094506 (2006).

${ }^{16}$ B. Ruhstaller, S. A. Carter, S. Barth, H. Riel, W. Riess, and J. C. Scott, J. Appl. Phys. 89, 4575 (2001).

${ }^{17}$ E. Purcell, "Spontaneous emission probabilities at radio frequencies," Phys. Rev. 69(1-2), 681 (1946).

${ }^{18}$ W. Brütting, S. Berleb, and A. G. Mückl, Org. Electron. 2, 1 (2001).

${ }^{19}$ S. Nowy, "Understanding losses in OLEDs: Optical device simulation and electrical characterization using impedance spectroscopy," Ph.D. dissertation (University of Augsburg, 2010).

${ }^{20}$ S. Nowy, W. Ren, J. Wagner, J. A. Weber, and W. Brütting, Proc. SPIE 7415, 74150G (2009).

${ }^{21}$ Y. Noguchi, Y. Miyazaki, Y. Tanaka, N. Sato, Y. Nakayama, T. D. Schmidt, W. Brütting, and H. Ishii, J. Appl. Phys. 111, 114508 (2012).

${ }^{22}$ Physics of Organic Semiconductors, edited by W. Brütting (Wiley-VCH, Berlin, 2012).

${ }^{23}$ Y. Noguchi, H. Kim, R. Ishino, K. Goushi, C. Adachi, Y. Nakayama, and H. Ishii, Org. Electron. 17, 184-191 (2015).
${ }^{24}$ T. Tsutsui, E. Aminaka, C. P. Lin, and D. U. Kim, Philos. Trans. Soc. A 355, 801 (1997).

${ }^{25}$ M. A. Baldo, D. F. O’Brien, Y. You, A. Shoustikov, S. Sibley, M. E. Thompson, and S. R. Forrest, Nature 395, 151 (1998).

${ }^{26}$ J. S. Wilson, A. S. Dhoot, A. J. A. B. Seeley, M. S. Khan, A. Kohler, and R. H. Friend, Nature 413, 828 (2001).

${ }^{27}$ Y. Sun, N. C. Giebink, H. Kanno, B. Ma, M. E. Thompson, and S. R. Forrest, Nature 440, 908 (2006).

${ }^{28}$ Y. Luo and H. Aziz, Adv. Funct. Mater. 20, 1285 (2010).

${ }^{29}$ D. Yokoyama, Y. Park, B. Kim, S. Kim, Y.-J. Pu, J. Kido, and J. Park, Appl. Phys. Lett. 99, 123303 (2011).

${ }^{30}$ Y.-J. Pu, G. Nakata, F. Satoh, H. Sasabe, D. Yokoyama, and J. Kido, Adv. Mater. 24, 1765 (2012).

${ }^{31}$ H. Sternlicht, G. C. Nieman, and G. W. Robinson, J. Chem. Phys. 38, 1326 (1963).

${ }^{32}$ A. Endo, K. Sato, K. Yoshimura, T. Kai, A. Kawada, H. Miyazaki, and C. Adachi, Appl. Phys. Lett. 98, 083302 (2011).

${ }^{33}$ H. Uoyama, K. Goushi, K. Shizu, H. Nomura, and C. Adachi, Nature 492, 234-238 (2012).

${ }^{34}$ T. Nakagawa, S.-Y. Ku, K.-T. Wong, and C. Adachi, Chem. Commun. 48, 9580 (2012)

${ }^{35}$ T. D. Schmidt, D. S. Setz, M. Flämmich, J. Frischeisen, D. Michaelis, C. Mayr, A. F. Rausch, T. Wehlus, B. J. Scholz, T. C. G. Reusch, N. Danz, and W. Brütting, Appl. Phys. Lett. 103, 093303 (2013).

${ }^{36}$ C. Mayr, S. Y. Lee, T. D. Schmidt, T. Yasuda, C. Adachi, and W. Brütting, Adv. Funct. Mater. 24, 5232-5239 (2014).

${ }^{37}$ S. Nowy, B. C. Krummacher, J. Frischeisen, N. A. Reinke, and W. Brütting, J. Appl. Phys. 104, 123109 (2008).

${ }^{38}$ D. S. Setz, T. D. Schmidt, M. Flämmich, S. Nowy, J. Frischeisen, B. C. Krummacher, T. Dobbertin, K. Heuser, D. Michaelis, N. Danz, W. Brütting, and A. Winnacker, J. Photon. Energy 1, 011006 (2011).

${ }^{39}$ T. D. Schmidt, D. S. Setz, M. Flämmich, J. Frischeisen, D. Michaelis, B. C. Krummacher, N. Danz, and W. Brütting, Appl. Phys. Lett. 99, 163302 (2011).

${ }^{40}$ T. D. Schmidt, M. Flämmich, B. J. Scholz, D. Michaelis, C. Mayr, N. Danz, and W. Brütting, Proc. SPIE 8435, 843513 (2012).

${ }^{41}$ T. D. Schmidt, B. J. Scholz, C. Mayr, and W. Brütting, IEEE J ST Quantum Electron. 19, 1 (2013).

${ }^{42}$ M. Flämmich, J. Frischeisen, D. S. Setz, D. Michaelis, B. C. Krummacher, T. D. Schmidt, W. Brütting, and N. Danz, Org. Electron. 12, 1663 (2011).

${ }^{43}$ W. Brütting, J. Frischeisen, T. D. Schmidt, B. J. Scholz, and C. Mayr, Phys. Status Solidi A 210, 44 (2013).

${ }^{44}$ S. Reineke, K. Walzer, and K. Leo, Phys. Rev. B 75, 125328 (2007).

${ }^{45}$ D. Y. Kondakov, W. C. Lenhart, and W. F. Nichols, J. Appl. Phys. 101, 024512 (2007).

${ }^{46}$ D. Y. Kondakov, J. R. Sandifer, C. W. Tang, and R. H. Young, J. Appl. Phys. 93, 1108 (2003).

${ }^{47}$ M. Kuik, L. Koster, A. Dijkstra, G. Wetzelaer, and P. Blom, Org. Electron. 13, 969 (2012).

${ }^{48}$ M. Kuik, H. T. Nicolai, M. Lenes, G.-J. A. H. Wetzelaer, M. Lu, and P. W. M. Blom, Appl. Phys. Lett. 98, 093301 (2011).

${ }^{49}$ M. Kuik, L. J. A. Koster, G. A. H. Wetzelaer, and P. W. M. Blom, Phys. Rev. Lett. 107, 256805 (2011).

${ }^{50}$ G. A. H. Wetzelaer, M. Kuik, H. T. Nicolai, and P. W. M. Blom, Phys. Rev. B 83, 165204 (2011).

${ }^{51}$ R. Coehoorn, H. van Eersel, P. Bobbert, and R. Janssen, Adv. Funct. Mater. 25, 2024 (2015).

${ }^{52}$ C. Fery, B. Racine, D. Vaufrey, H. Doyeux, and S. Cina, Appl. Phys. Lett. 87, 213502 (2005).

${ }^{53}$ S. Berleb, W. Brütting, and G. Paasch, Org. Electron. 1, 41 (2000).

${ }^{54}$ E. Ito, Y. Washizu, N. Hayashi, H. Ishii, N. Matsuie, K. Tsuboi, Y. Ouchi, Y. Harima, K. Yamashita, and K. Seki, J. Appl. Phys. 92, 7306 (2002).

${ }^{55}$ W. Riess, H. Riel, T. Beierlein, W. Brütting, P. Müller, and P. Seidler, IBM J. Res. Develop. 45, 77 (2001).

${ }^{56}$ J. Kalinowski, W. Stampor, J. Mężyk, M. Cocchi, D. Virgili, V. Fattori, and P. Di Marco, Phys. Rev. B 66, 235321 (2002). 